\section{Development of an information booklet for small animal pain management clinic clients}

\section{Tate Preston, Ian Self}

The University of Nottingham, Nottingham, United Kingdom

\section{OBJECTIVES}

Ascertain from veterinary clients what information would be useful in the management of their dog's pain.

Produce an information booklet based on this information.

Subsequently improve the welfare of pet dogs presented with painful conditions to veterinary surgeons.

\section{METHODS}

Semi-structured interviews were held with clients attending a referral pain clinic and a hydrotherapy centre regarding what information would be useful for them and their attitudes towards dog pain. Interviews were recorded and results analysed using NVivo (qualitative data analysis software). A booklet was produced answering the main questions raised by the owners, together with background information provided by veterinary analgesia specialists.

\section{RESULTS}

166 codes were generated from 7844 references from which 6 themes were identified; Variety of treatment options, Dog pain is comparable to human pain, Not being normal shows the dog is in pain, Owner responsibility, Trust, Understanding your dog and its pain.

Pain Treatment was mentioned 652 times (the highest number of references).

\section{STATEMENT (CONCLUSIONS)}

Participants believe a combination of treatments work best including therapies, pharmaceuticals, surgery and environmental management. Owners feel a responsibility as advocates of their dog's quality of life. Owners need to be able to trust their vet/professional when treating and making decisions about their dog's pain, this includes knowing that they are competent. People want to be able to understand the signs of pain or lack of signs that their dog shows, they understand that dogs cannot communicate pain the same way as humans do.

\section{Acetabular fractures in 30 dogs: presentation, classification and management and short-term outcome}

\section{Victoria Roberts, Richard Meeson}

Royal Veterinary College, London, United Kingdom

\section{OBJECTIVES}

To describe the presentation, classification, management and short-term outcome of dogs with acetabular fractures.

\section{METHODS}

Retrospective analysis of the clinical data of dogs with at least one acetabular fracture between January 2010 and March 2018.

\section{RESULTS}

30 dogs with 32 radiographically confirmed acetabular fractures met inclusion criteria, of which 27 had confirmed/ suspected vehicular trauma. Mean age was 34 months (range: 5-120), mean weight was $10.7 \mathrm{~kg}$ (range: $2.66-$ 27.55), 14/30 were entire, $17 / 30$ were female.

The majority were unilateral (28/32) and simple (19/32). Most commonly seen in the mid (20), or caudal acetabulum (10), rarely in the cranial quadrant (2). $28 / 30$ had additional orthopaedic injuries and 2 were euthanised due to co-morbidities. 22/32 underwent surgical stabilisation; acetabular plate $(A P)(n=8)$, femoral head and neck excision (FHNE) $(n=5)$, screws, wires and polymethylmethacrylate $(S W-P M M A)(n=3)$, dynamic compression plate $(n=1), A P$ and SW-PMMA $(n=1)$, FHNE and locking compression plate $(n=1), A P$ and wire $(n=1)$, FHNE and wire $(n=1)$, screws and wire $(n=1)$. $8 / 32$ were managed conservatively, with 4 undergoing other pelvic surgery. 27 dogs (28 fractures) survived to discharge. 18 dogs had follow-up with 11 lameness scores recorded, mean 3.7/10 (range: 0-10). 15 fractures had radiographs after 28 days, all showed evidence of bone healing. Complications seen were: 1 had FHNE 9 days post-operatively due to subsequent coxofemoral luxation, 1 acetabular plate failed not requiring revision surgery.

\section{STATEMENT (CONCLUSIONS)}

Canine acetabular fractures commonly occur secondary to vehicular trauma and are usually unilateral, simple and occupy the mid or caudal acetabulum. The majority were surgically managed and complications were rare. 